Pacific Journal of Mathematics

RINGS WHOSE KERNEL FUNCTORS ARE LINEARLY

ANA M. VIOLA-PRIOLI AND JoRGE VIOLA-PRIO 


\title{
RINGS WHOSE KERNEL FUNCTORS ARE LINEARLY ORDERED
}

\section{ANa M. dA Rocha de Viola-Prioli and Jorge E. Viola-Prioli}

\begin{abstract}
Valuation domains have been extended in the non-commutative case by several authors, giving rise to the so called generalized valuation rings, that is, rings whose lattice of right ideals is linearly ordered by inclusion. We propose here the study of rings whose lattice of kernel functors is linearly ordered and we indicate throughout this article similarities between them and valuation rings. In addition, the rings presented here include generalized valuation rings and coincide with them when commutativity is assumed. They therefore provide a new non-commutative analogue of valuation rings.

Unlike the generalized valuation rings, the rings we study enjoy properties that transfer nicely to matrix rings thus enabling us to treat questions in a broader context.

Finally, a semigroup structure imposed on the lattice of kernel functors is analyzed and the article concludes by examining when that semigroup can be thought of as the semigroup of a valuation ring.
\end{abstract}

Preliminaries. All rings occurring are associative and possess unity, which is preserved under subrings and ring homomorphisms. Unless otherwise stated all modules are unitary right modules. We let $\mathscr{M}_{R}$ denote the category of right $R$-modules.

For any module $M$ we let $E(M)$ stand for an injective hull of $M$. Hence $M$ is large in $E(M)$ and $E(M)$ is injective. If $M$ is a module, $N$ a submodule of $M$ and $S$ a nonempty subset of $M$ we let $(N: S)$ denote the right ideal $\{r \in R ; S r \subset N\}$. When no danger of confusion arises we will simply write $(N: S)$.

The term ideal is reserved to be used for two-sided ideals only. Consequently, a ring is simple if it has exactly two ideals.

Notation and terminology concerning kernel functors, (topologizing) filters of right ideals, etc., will follow Goldman [4] and Stenstrom [9] with which familiarity is assumed.

The class of all objects of $\mathscr{M}_{R}$ which are torsion with respect to a given kernel functor is closed under taking submodules, homomorphic images and arbitrary direct sums, conditions that characterize what we will call a torsion class throughout this paper. There is a one to one correspondence between kernel functors, filters of right ideals and torsion classes. 
We let $\mathscr{L}$ denote the filter of large (or essential) right ideals of $R$ and $Z$ its associated kernel functor; consequently $Z(M)$ is the singular submodule of $M$.

The idempotent filter of dense (or rational) right ideals of $R$ is indicated by $\mathscr{D}$; thus $\mathscr{D} \subset \mathscr{L}$ and they coincide precisely when $Z(R)=0$. In this paper $\mathbf{I}(R)$ stands for the set of idempotent kernel functors while $Q_{\sigma}(R)$ denotes the ring of right quotients of $R$ with respect to $\sigma$, if $\sigma$ belongs to $\mathbf{I}(R)$.

For every module $M$, the direct sum of $n$ copies of $M$ is denoted by $M^{(n)}$. Also, $\operatorname{Soc}(M)$ indicates the sum of all the simple submodules of $M$, or (0) when $M$ contains no such submodules.

$R$ is a (right) $V$-ring if every simple $R$-module is injective. (See Faith [2].) A module $M$ is called proper cyclic if it is cyclic and non-isomorphic to $R$. As in [2] $R$ is a PCI-ring whenever its proper cyclic modules are injective.

By a valuation ring we mean a commutative ring whose ideals are linearly ordered by inclusion. If in addition the ring has no zero-divisors it is called a valuation domain.

Any unexplained terminology can be found, for instance, in [2] or [3].

$\mathbf{K}(R)$ linearly ordered. Let $\mathbf{K}(R)$ denote the set of all the kernel functors of the ring $R$; it is a complete lattice because there is a partial ordering in which $\sigma \leq \nu$ means $\sigma(M) \subset \nu(M)$ for all modules $M$, and every family $\left\{\sigma_{i}\right\}$ of kernel functors has a supremum and an infimum, defined by $\sup \left\{\sigma_{i}\right\}=\sigma^{*}$, where $\sigma^{*}(M)=\sum \sigma_{i}(M)$ for every $M \in \mathscr{M}_{R}$ $\inf \left\{\sigma_{i}\right\}=\sigma_{*}$, where $\sigma_{*}(M)=\bigcap \sigma_{i}(M)$ for every $M \in \mathscr{M}_{R}$. We treat in this article the rings $R$ such that $\mathbf{K}(R)$ is linearly ordered (under the ordering defined above).

PROPOSITION 1. The following conditions are equivalent:

(a) $\mathbf{K}(R)$ is linearly ordered.

(b) For every $I_{R} \subset R$ and $J_{R} \subset R$, there exists a finite set $X \subset R$ such that $I \supset(J: X)$ or $J \supset(I: X)$.

(c) For every $I_{R} \subset R$ and $J_{R} \subset R$, there exists a natural number $n=$ $n(I, J)$ such that either $R / I$ is an epimorphic image of a submodule of $(R / J)^{(n)}$, or $R / J$ is an epimorphic image of a submodule of $(R / I)^{(n)}$.

Proof. (a) $\Rightarrow$ (b) Set $\mathscr{F}(I)=\left\{U_{R} \subset R\right.$; there exists a finite set $S \subset R$ such that $(I: S) \subset U\}$ and define analogously $\mathscr{F}(J)$. It is easy to verify that they are topologizing filters and therefore we can assume $\mathscr{F}(I) \subset$ $\mathscr{F}(J)$. Since obviously $I \in \mathscr{F}(I),(\mathrm{b})$ follows. 
(b) $\Rightarrow$ (c) Assume $J \supset(I: X)$ where $X=\left\{x_{1}, \ldots, x_{n}\right\} \subset R$, and define $f: R \rightarrow(R / I)^{(n)}$ such that $f(r)=\left(\bar{x}_{1}, \ldots, \bar{x}_{n}\right) r$. Since kernel $f=$ $(I: X) \subset J$, it follows that $f(R) \cong R /(I: X)$ and $R /(I: X)$ maps onto $R / J$. Therefore $R / J$ is an epimorphic image of $f(R)$, which is a submodule of $(R / I)^{(n)}$.

(c) $\Rightarrow$ (a) Let $\sigma$ and $\nu$ be elements of $\mathbf{K}(R)$ such that $\sigma \nless \nu$. Then there exists a $\sigma$-torsion cyclic module $R / I$ which is not $\nu$-torsion. Let $\mathscr{F}$ be the filter associated with $\nu$ and $J \in \mathscr{F}$. Properties of torsion classes guarantee that no submodule of $(R / J)^{(n)}$ can map onto $R / I$, and thus by hypothesis $R / J$ is an epimorphic image of $(R / I)^{(n)}$, for a certain natural number $n$. We conclude that $R / J$ is $\sigma$-torsion, for every $J \in \mathscr{F}$ and so $\nu \leq \sigma$.

A further step in building this kind of ring is provided by the next result.

Proposition 2. Let $R$ be a ring such that $\mathbf{K}(R)$ is linearly ordered and let $S$ be a ring. Then

(a) If $S$ is an epimorphic image of $R, \mathbf{K}(S)$ is linearly ordered.

(b) IF $S$ is Morita equivalent to $R, \mathbf{K}(S)$ is linearly ordered.

Proof. (a) Let $g: R \rightarrow S$ be the given epimorphism and let $g(I)$ and $g(J)$ be right ideals of $S$. We may assume kernel $g \subset I \cap J$ and also $I \supset(J: X)$ for a certain finite set $X \subset R$. It follows that if $g(r) \in$ $(g(J)): g(s))$ then $r \in(J: X)$ and therefore $g(r) \in g(I)$. Thus $(g(J) \stackrel{\leftrightarrow}{S} g(X)) \subset g(I)$ and $\mathbf{K}(S)$ is linearly ordered.

(b) Given a category equivalence $\psi: \mathscr{M}_{R} \rightarrow \mathscr{M}_{S}$ and torsion classes $\mathscr{A}$ and $\mathscr{B}$ in $\mathscr{M}_{S}$, define $\mathscr{A}^{\prime}=\left\{M_{R} ; \psi(M) \in \mathscr{A}\right\}$ and $\mathscr{B}^{\prime}=\left\{M_{R} ; \psi(M)\right.$ $\in \mathscr{B}\}$. Since $\mathscr{A}^{\prime}$ and $\mathscr{B}^{\prime}$ are torsion classes in $\mathscr{M}_{R}$, we may assume $\mathscr{A}^{\prime} \subset \mathscr{B}^{\prime}$. It is easily checked now that $\mathscr{A} \subset \mathscr{B}$.

COROllaRy 3. If $\mathbf{K}(R)$ is linearly ordered, then $\mathbf{K}\left(M_{n \times n}(R)\right)$ is linearly ordered.

For every ideal $I$ of $R$ and for every set $X \subset R$ we have $I \subset(I: X)$. According to Proposition 1 we conclude:

COROllary 4. If $\mathbf{K}(R)$ is linearly ordered the ideals of $R$ are linearly ordered by inclusion.

We will next consider conditions to ensure the validity of the converse of Corollary 4. Similar results (by taking only right sided ideals) will also 
be formulated. Let $I$ be an ideal of $R$ and let us define the following collections:

$$
\begin{aligned}
& \mathscr{F}(I)=\left\{K_{R} \subset R ; I \subsetneq K\right\} \quad \text { if } I \varsubsetneqq R, \\
& \overline{\mathscr{F}}(I)=\left\{K_{R} \subset R ; I \subset K\right\} .
\end{aligned}
$$

LEMMA 5. If the right ideals of $R$ are linearly ordered by inclusion and $I$ is a proper ideal of $R$, then $\mathscr{F}(I)$ and $\overline{\mathscr{F}}(I)$ are topologizing filters.

Proof. $\overline{\mathscr{F}}(I)$ is always a filter. With regards to $\mathscr{F}(I)$ it suffices to prove that if $X \in \mathscr{F}(I)$ and $r \in R$ then $(X: r) \in \mathscr{F}(I)$. Assume $(X: r)$ $=I$. By hypothesis either $r R \subset X$ or $X \subset r R$. If $r R \subset X$ then $R \subset(X: r)$ $=I$, a contradiction. Therefore we must have $X \subset r R$. Pick $x \in X$ such that $x \notin I$, and $\lambda \in R$ such that $x=r \lambda$. It follows that $\lambda \in(X: r)$ and so $x \in I$, a contradiction. We conclude $(X: r) \supsetneqq I$ and thus $\mathscr{F}(I)$ is a filter, as asserted.

LEMMA 6. If the right ideals of $R$ are linearly ordered by inclusion, for every filter $\mathscr{F}$ there exists an ideal I such that either $\mathscr{F}=\mathscr{F}(I)$ or $\mathscr{F}=\overline{\mathscr{F}}(I)$.

Proof. Given the filter $\mathscr{F}$, define $I_{0}=\bigcap J$, taken for all $J \in \mathscr{F}$. Since $I_{0}$ is an ideal of $R$, if $I_{0} \in \mathscr{F}$ then $\mathscr{F}=\overline{\mathscr{F}}\left(I_{0}\right)$. Let us show now that if $I_{0} \notin \mathscr{F}$ then $\mathscr{F}=\mathscr{F}\left(I_{0}\right)$. To that end, given $J \in \mathscr{F}\left(I_{0}\right)$ we must have $J \supsetneqq I_{0}$ and so there exists $H \in \mathscr{F}$ such that $J \not \subset H$. But then $H \subset J$, by hypothesis, and $J$ belongs to $\mathscr{F}$. Therefore $\mathscr{F}\left(I_{0}\right) \subset \mathscr{F}$ which suffices to show that $\mathscr{F}=\mathscr{F}\left(I_{0}\right)$.

COROLlaRY 7. If the right ideals of $R$ are linearly ordered by inclusion, then $\mathbf{K}(R)$ is linearly ordered.

Proof. Given $I$ and $J$ ideals of $R$ such that $I \subset J$, it follows that $\mathscr{F}(J) \subset \overline{\mathscr{F}}(J) \subset \mathscr{F}(I) \subset \overline{\mathscr{F}}(I)$. We make use now of Lemma 6 to infer that filters (and so, kernel functors) are linearly ordered.

The converse of last result is not valid, as we may see by taking $R=M_{2 \times 2}(F)$, where $F$ is a field. According to Corollary $3 \mathbf{K}(R)$ is linearly ordered; however the right ideals $\left[\begin{array}{ll}1 & 0 \\ 0 & 0\end{array}\right] R$ and $\left[\begin{array}{ll}0 & 0 \\ 0 & 1\end{array}\right] R$ are not comparable. 
Proposition 8. If either $R$ is right artinian or every right ideal of $R$ is two-sided, then $\mathbf{K}(R)$ is linearly ordered if and only if the ideals of $R$ are linearly ordered by inclusion.

Proof. (a) Assume $R_{R}$ is artinian. Let $\sigma$ and $\nu$ be elements of $\mathbf{K}(R), \mathscr{F}$ and $\mathscr{G}$ their corresponding filters and let $I_{R}$ and $J_{R}$ be minimal elements of $\mathscr{F}$ and $\mathscr{G}$ respectively. It follows that $I$ and $J$ are ideals, $\mathscr{F}=\overline{\mathscr{F}}(I)$ and $\mathscr{G}=\overline{\mathscr{F}}(J)$. If the ideals of $R$ are linearly ordered by inclusion, so are the filters, as $I \subset J$ implies $\overline{\mathscr{F}}(I) \supset \overline{\mathscr{F}}(J)$. Therefore, $\mathbf{K}(R)$ is linearly ordered.

(b) If every right ideal is two-sided, this assertion reduces to Corollary 7 .

As a consequence we have

COROllary 9. Let $R$ be a commutative ring. Then $\mathbf{K}(R)$ is linearly ordered if and only if $R$ is a valuation ring.

According to this result, the rings under consideration can be viewed as non-commutative analogues of valuation rings. We aim therefore to providing analogies between these two kinds of rings. Next statement applies to any given ring.

Proposition 10. $\mathbf{K}(R)-\{\infty\}$ has maximal elements. Dually, $\mathbf{K}(R)$ - $\{\mathbf{0}\}$ has minimal elements.

Proof. Set $A=\mathbf{K}(R)-\{\infty\}$. Given an ascending chain $\left\{\sigma_{i}\right\}$ in $A$ denote $\sup \left\{\sigma_{i}\right\}$ by $\sigma^{*}$. If $\sigma^{*}=\infty$, then $1 \in \sigma^{*}(R)=\sum \sigma_{i}(R)$. Hence there exists an index $k$ such that $1 \in \sigma_{k}(R)$ and thus $\sigma_{k}=\infty$, a contradiction. Accordingly, $\sigma^{*} \in A$ and the usual Zorn's Lemma argument furnishes a maximal element of $A$.

On the other hand, let $S$ be a simple module, set $\mathscr{C}=\left\{\oplus M_{i}\right.$; $\left.M_{1} \cong S\right\}$ and let $\nu$ be the kernel functor associated with the torsion class $\mathscr{C}$; we clearly have $\nu>\mathbf{0}$. Next assume there exists $\sigma \in \mathbf{K}(R)$ such that $\mathbf{0}<\sigma \leq \nu$ and let us show that $\sigma=\nu$. To that end pick a non-zero $\sigma$-torsion cyclic module $M$ and a simple module $T$ onto which $M$ is mapped. Therefore $T$ is $\sigma$-torsion and so $T$ is $\nu$-torsion. Hence $T \cong \oplus S$ which implies $T \cong S$. We conclude that $S$ is $\sigma$-torsion, which forces every element of $\mathscr{C}$ to be $\sigma$-torsion, that is, $\nu \leq \sigma$. It follows that $\nu$ is a minimal element, as required. 
As a consequence of the proof just given, we now obtain:

COROllaRY 11. If $\mathbf{K}(R)$ is linearly ordered, then $\mathbf{K}(R)-\{\infty\}$ has a maximum and $\mathbf{K}(R)-\{\mathbf{0}\}$ has a minimum. Moreover, there exists a unique simple $R$-module (up to isomorphisms) and the minimum of $\mathbf{K}(R)$ $\{\boldsymbol{0}\}$ is Soc.

As to Corollary 3, we can now show that its converse is not always valid. In fact, [6] contains examples of simple rings $R$ having infinitely many non-isomorphic simple modules. Although clearly the ideals of $R$ are linearly ordered (by inclusion), the elements of $\mathbf{K}(R)$ are not.

Let $\mathbf{K}(R)$ be linearly ordered and let $\sigma_{\max }$ denote the maximum of $\mathbf{K}(R)-\{\infty\}$.

In the commutative case, our rings reduce to valuation rings, according to Corollary 9. Clearly, in this case the filter associated with $\sigma_{\max }$ coincides with $\{I \subset R ; I \neq(0)\}=\mathscr{F}(0)$ and thus, for every $M \in \mathscr{M}_{R}$, we have $\sigma_{\max }(M)=\{m \in M$; there exists $0 \neq r \in R$ such that $m r=0\}=$ $Z(M)$. However, the situation changes in the non-commutative case and the description of $\sigma_{\max }$ turns out to be more complicated. It is worth emphasizing that the minimum of $\mathbf{K}(R)-\{\mathbf{0}\}$ is Soc whether $R$ is commutative or not.

At any rate, if $\mathbf{K}(R)$ is linearly ordered, then Soc $\leq \sigma_{\max }$ except when $\mathbf{K}(R)=\{\mathbf{0}, \infty\}$. But in this case Soc $=\infty$, every module is semisimple and $R \cong M_{n \times n}(D)$, for a certain division ring $D$.

We recall that $R$ is an absolutely torsion free ring (ATF, for short) whenever $\sigma(R)=(0)$ for every $\sigma \in \mathbf{K}(R)-\{\infty\}$. (See [7], [10].)

Proposition 12. Let $\mathbf{K}(R)$ be linearly ordered and assume $\sigma_{\max }$ is idempotent. Then, $R$ is an ATF-ring and $\sigma_{\max }(M)=Z(M)$ for every $M \in \mathscr{M}_{R}$.

Proof. To tackle ATF-ness first, it will suffice showing that $\sigma_{\max }(R)$ $=(0)$, since $\sigma \leq \sigma_{\max }$ for every $\sigma<\infty$. Write $I=\sigma_{\max }(R)$ and assume $I \neq(0)$. Then $\nu(M)=\{m \in M ; m I=(0)\}$ defines a kernel functor, and clearly $\nu<\infty$. Therefore $\nu \leq \sigma_{\max }$ and so $R / I$ is a $\sigma_{\max }$-torsion module. But so is $I$, and the idempotency of $\sigma_{\max }$ guarantees that $R$ is $\sigma_{\max }$-torsion, that is, $\sigma_{\max }=\infty$, a contradiction. That proves $R$ is an ATF-ring. Next, as in [7] we obtain $\sigma_{\max }=\tau_{E(R)}$ which shows that the filter associated with $\sigma_{\max }$ is $\mathscr{D}$. Since $R$ is a non-singular ring, it follows that $\mathscr{D}=\mathscr{L}$ and therefore $\sigma_{\max }(M)=Z(M)$ for every $M \in \mathscr{M}_{R}$. 
It is well known that the localizations of any valuation domain are linearly ordered under inclusion. The non-commutative analogous result will be proved next. Let us remark that a commutative ring is ATF if and only if it is a domain. (See [10].)

Proposition 13. If $R$ is an ATF-ring and $\mathbf{K}(R)$ is linearly ordered, then its localizations are linearly ordered.

Proof. Since $R$ is ATF it is a non-singular ring and therefore every ring of right quotients of $R$ is a subring of the maximal ring of quotients of $R$, which in this case turns out to be $E(R)$. (See [9].) If $A$ is a localization of $R$, according to [9] there exists $\sigma \in \mathbf{I}(R)-\{\infty\}$ such that $A=Q_{\sigma}(R)$ and therefore $R \subset A \subset E(R)$. Now, by definition of ring of quotients we must have $A / R=Q_{\sigma}(R) / R=\sigma(E(R) / R)$. The same argument applies to any other given localization $B$, allowing us to conclude that $B / R=Q_{\lambda}(R) / R=\lambda(E(R) / R)$ for a certain idempotent $\lambda$. Since by hypothesis $\sigma \leq \lambda$, say, we infer that $A \subset B$, and the conclusion follows.

Every valuation ring is local and, if not a field, has a nilpotent socle. Next results show that this generalizes quite nicely in our non-commutative context. In what follows, $J(R)$ stands for the Jacobson radical of $R$.

Proposition 14. If $\mathbf{K}(R)$ is linearly ordered, then

(a) $J(R)$ contains every proper ideal of $R$.

(b) If $U=\operatorname{Soc}(R)$, then either $U^{2}=(0)$ or $U=R$ (and so $R \cong$ $M_{n \times n}(D)$ in this case).

Proof. (a) Given a proper ideal $I$, we will show that $I \subset T$ for every maximal right ideal $T$. Pick a maximal right ideal $P$ containing $I$. According to Corollary 11 there exists an isomorphism $f: R / T \rightarrow R / P$. Since $I$ is two-sided, it annihilates $R / P$ and hence it annihilates $R / T$. Therefore, $I \subset T$, as stated.

(b) Assume $U \neq R$. Let us define an element of $\mathbf{K}(R)-\{\mathbf{0}\}$ by setting $\nu(M)=\{m \in M ; m U=(0)\}$. Then, by Corollary 11, Soc $\leq \nu$ so that $U=\operatorname{Soc}(R) \subset \nu(R)$ which implies $U^{2} \subset \nu(R) U=(0)$, as claimed.

COROllaRy 15. Let $\mathbf{K}(R)$ be linearly ordered. Then the following assertions hold:

(a) If $H$ is an ideal of $R$, either $H$ is right essential in $R$ or $H^{2}=(0)$.

(b) If $J(R)$ is not essential in $R$, then $J(R)=(0)$. 
Proof. (a) Assume $H$ is non vanishing. If $H_{R}$ is not essential pick a non-zero right ideal $I$ such that $H \cap I=(0) . H$ is two-sided and so either $R I \subset H$ or $H \subset R I$. But $R I \subset H$ implies $I \subset H$ and so $I=(0)$, a contradiction. Hence, $H \subset R I$ holds and we readily have $H^{2} \subset(R I) H=$ $R(I H)=(0)$.

(b) Suppose $J(R)$ is not essential. If in addition $J(R) \neq(0)$ we proceed as in (a) with $H=J(R)$. This time, however, the argument yields necessarily $R I \subset J(R)$, as Proposition 14 applies. As before, this is a contradiction.

One evidence of the ubiquity of simple rings when kernel functors are dealt with, is given next.

CorollaRY 16. Let $\mathbf{K}(R)$ be linearly ordered. If $R$ is either (von Neumann) regular, or a $V$-ring, or primitive, then $R$ is a simple ring.

Proof. In any of these cases, $J(R)=(0)$. The conclusion follows, by Proposition 14.

In valuation rings every non-zero idempotent ideal has a nilpotent annihilator. It is natural to try to extend this fact to idempotent kernel functors.

Proposition 17. Assume $\mathbf{K}(R)$ is linearly ordered. Then

(a) $\sigma(R)^{2}=(0)$ for every $\boldsymbol{\sigma} \in \mathbf{I}(R)-\{\infty\}$.

(b) If $R$ is semiprime, $\sigma(R)=(0)$ for every $\sigma \in \mathbf{I}(R)-\{\infty\}$.

Proof. (a) Write $I=\sigma(R)$. We proceed as in Proposition 12, that is, we define $\nu(M)=\{m \in M ; m I=(0)\}$ for every $M \in \mathscr{M}_{R}$. Thus, $\nu \in$ $\mathbf{K}(R)-\{\infty\}$ and if $\nu \leq \sigma, R / I$ turns out to be $\sigma$-torsion. But $I$ is $\sigma$-torsion and $\sigma$ is idempotent, which implies that $R$ is $\sigma$-torsion, a contradiction since $\sigma \neq \infty$. Therefore we must have $\sigma<\nu$, so that $I=$ $\sigma(R) \subset \nu(R)$. Finally, $I^{2} \subset \nu(R) I=(0)$, as stated.

We recall that domains (whether commutative or not) are ATF-rings; the following result shows that the converse is valid for some of the rings treated in this article.

Proposition 18. Assume the right ideals of $R$ are linearly ordered by inclusion. Then $R$ is ATF if and only if $R$ is a domain. 
Proof. If $R$ is ATF we know $\sigma_{\max }(R)=(0)$. Moreover, by Lemma 6 the filter associated with $\sigma_{\max }$ is $\mathscr{F}(0)=\left\{I_{R} ;(0) \neq I \subset R\right\}$. Then, if $a b=0$ and $b \neq 0$ we have $b R \in \mathscr{F}(0)$ and consequently $a \in \sigma_{\max }(R)=$ $(0)$.

Proposition 19. If $\mathbf{K}(R)$ is linearly ordered then the center of $R$ is a local ring.

Proof. Let $A$ be the center of $R$. We proceed to prove that given $a$ and $b$ non-units of $A, a+b$ is not a unit in $A$. Suppose $a+b$ is invertible in $A$ and pick $s \in A$ such that $(a+b) s=1$. Write $a^{\prime}=a s$ and $b^{\prime}=b s$ and consider $a^{\prime} R$ and $b^{\prime} R$. Since they are two-sided, by virtue of Corollary 4 we may assume $a^{\prime} R \subset b^{\prime} R$, and so $a^{\prime}=b^{\prime} r$, for a certain $r \in R$. We claim $r \in A$. In fact, given $r^{*} \in R$, we observe that

$$
\begin{aligned}
b^{\prime}\left(r r^{*}-r^{*} r\right) & =b^{\prime} r r^{*}-b^{\prime} r^{*} r=a^{\prime} r^{*}-r^{*} b^{\prime} r \\
& =r^{*} a^{\prime}-r^{*} b^{\prime} r=r^{*} a^{\prime}-r^{*} a^{\prime}=0 .
\end{aligned}
$$

Then, writing $z$ for $r r^{*}-r^{*} r$, we have $b^{\prime} z=0$. Moreover, $a^{\prime} z=b^{\prime} r z=$ $r b^{\prime} z=0$. Since $a^{\prime}+b^{\prime}=1$ we infer that $z=0$, that is, $r r^{*}=r^{*} r$. We conclude that $r \in A$, as claimed. Finally,

$$
1=a^{\prime}+b^{\prime}=b^{\prime} r+b^{\prime}=b(s(r+1))
$$

which means $b$ is a unit of $A$, the desired contradiction.

By [4] for every ring $R$ and for every $\sigma \in \mathbf{K}(R)$ one can construct $\bar{\sigma} \in \mathbf{I}(R), \bar{\sigma} \geq \sigma$ such that $\bar{\sigma}(M)=(0)$ if and only if $\sigma(M)=(0)$. We furnish the following two examples related with this fact:

EXAmple 1 . There exists a ring $R$ such that $\mathbf{I}(R)$ is linearly ordered but $\mathbf{K}(R)$ is not.

In fact, let $F$ be a field and let $A$ be the commutative algebra $F[x, y]$, together with the condition $x^{3}=y^{3}=0 . A$ is an artinian ring and $P=(x, y)$ is a prime ideal of $A$. Hence the ring $R=A_{P}$ is local artinian and therefore it is perfect and has a unique simple module. According to [11] we have $\mathbf{I}(R)=\{\mathbf{0}, \infty\}$; however $R$ is not a valuation ring since $(x)$ and $(y)$ are not comparable in $R$, and thus $\mathbf{K}(R)$ is not linearly ordered.

EXAMPLE 2. Let us construct a ring $R$ such that $\mathbf{K}(R)$ is a linearly ordered infinite lattice, and $\mathbf{I}(R)$ is finite. Let $F$ be a field and let $R$ be 
the commutative $F$-algebra generated by $\left\{x_{i}\right\}$, with $0 \leq i \leq 1$ and

$$
x_{i} x_{j}= \begin{cases}x_{i+j} & \text { if } i+j<1 \\ 0 & \text { if } i+j \geq 1\end{cases}
$$

It follows immediately that $x_{0}=1$ and $x_{1}=0$. Also $R$ is a local ring and its unique maximal ideal is the ideal $A_{0}$ generated by $\left\{x_{i} ; i>0\right\}$. Since every element of $A_{0}$ is nilpotent, $A_{0}$ is a nil-ideal. For every $i \in[0,1)$ set $A_{i}=\left\{x_{j} u ; u\right.$ is a unit and $\left.j>i\right\}, C_{i}=\left\{x_{j} u ; u\right.$ is a unit and $\left.j \geq i\right\}$ and set $A_{1}=C_{1}=(0)$. Clearly the ideals of $R$ are precisely the ideals $A_{i}$ and $C_{i}$ with $i \in[0,1]$, and whenever $0<i<s<1$ we have

$$
(0)=C_{1} \varsubsetneqq A_{s} \varsubsetneqq C_{s} \varsubsetneqq A_{i} \subsetneq C_{i} \varsubsetneqq A_{0} \varsubsetneqq C_{0}=R
$$

By Corollary 7, it follows that $\mathbf{K}(R)$ is linearly ordered. Now, the elements of $\mathbf{K}(R)$ are instantly surveyed: their associated filters are either $\mathscr{F}(I)$ or $\overline{\mathscr{F}}(I)$, according to Lemma 6 . We thus conclude $\mathbf{K}(R)$ is an infinite lattice. Next, we will exhibit the idempotent filters of $R$. Given such a filter $\mathscr{F}$, we consider the following two cases:

Case 1. $\mathscr{F}=\overline{\mathscr{F}}(I)$ for a certain ideal $I$. We must have $I=I^{2}$. If $I=C_{i}$ for a certain $i$, we infer that $C_{i}=C_{i} C_{i} \subset C_{2 i}$, and therefore $i=0$. This implies $I=C_{0}=R$ and consequently $\mathscr{F}=\{R\}$. Its corresponding kernel functor is $\mathbf{0}$. If $I=A_{i}$ for a certain $i$, a similar argument shows that $i$ vanishes, so that $I=A_{0}$ and $\mathscr{F}=\left\{A_{0}, R\right\}$. Its corresponding kernel functor is Soc.

Case 2. $\mathscr{F}=\mathscr{F}(I)$, for a certain ideal $I$ of $R$. If there exists $i$ such that $I=A_{i}$ we have $\mathscr{F}=\mathscr{F}\left(A_{i}\right)=\overline{\mathscr{F}}\left(C_{i}\right)$ which leads to the previous case. On the other hand, if there exists $i$ such that $I=C_{i}$, we must have $i>0$ and we can therefore pick $s>0$ such that $A_{s} \in \mathscr{F}$. Now, choose a natural number $n$ such that $n s>1$. Since every idempotent filter is multiplicative, it follows that $(0)=\left(A_{s}\right)^{n} \in \mathscr{F}$. We conclude that every ideal of $R$ belongs to $\mathscr{F}$, and the corresponding kernel functor is $\infty$.

What we obtained can be summarized as follows: $\mathbf{K}(R)$ is a linearly ordered infinite lattice whereas $\mathbf{I}(R)=\{\mathbf{0}$, Soc, $\infty\}$. Furthermore, it should be noticed that $\sigma_{\max }$ coincides with $Z$, although it is not idempotent.

Assume now $\mathbf{K}(R)$ is an infinite linearly ordered lattice. It will then contain a strictly increasing (or decreasing) infinite sequence of elements, a fact that in turn reflects on the lattice of right ideals of $R$, as will be shown next. 
Proposition 20. Assume $\mathbf{K}(R)$ is linearly ordered.

(a) If $\mathbf{K}(R)$ contains a strictly decreasing infinite sequence of elements, $R$ contains chains of right ideals of any given length.

(b) If $\mathbf{K}(R)$ contains a strictly increasing infinite sequence of elements, $R_{R}$ is not artinian.

Proof. (a) Given the chain of filters $\mathscr{F}_{1} \supsetneqq \mathscr{F}_{2} \supsetneqq \cdots$, pick $I_{1} \in \mathscr{F}_{1}-$ $\mathscr{F}_{2}$ and $I_{2} \in \mathscr{F}_{2}-\mathscr{F}_{3}$. Now set $K_{1}=I_{1} \cap I_{2}$ and $K_{2}=I_{2}$. It follows that $K_{1} \in \mathscr{F}_{1}-\mathscr{F}_{2}, K_{2} \in \mathscr{F}_{2}-\mathscr{F}_{3}$ and $K_{1} \subsetneq K_{2}$. Suppose we have constructed right ideals $K_{j} \in \mathscr{F}_{j}-\mathscr{F}_{j+1}$ for $j=1, \ldots, n$ in such a way that $K_{1} \varsubsetneqq K_{2} \varsubsetneqq \cdots \varsubsetneqq K_{n}$. Choose $X \in \mathscr{F}_{n+1}-\mathscr{F}_{n+2}$, set $K_{i}^{*}=K_{i} \cap X$ for $i \leq n$, and set $K_{n+1}^{*}=X$. Since $X \in \mathscr{F}_{j}$ for every $j \leq n$, we obtain $K_{J}^{*} \in \mathscr{F}_{j}$. Moreover, $K_{1}^{*} \subset K_{2}^{*} \subset \cdots \subset K_{n+1}^{*}$. If there exists $i \leq n$ such that $K_{1}^{*}=K_{i+1}^{*}$, we must have $K_{i}^{*} \in \mathscr{F}_{i+1}$, which implies $K_{i} \in \mathscr{F}_{i+1}$, a contradiction. We have shown that $\left\{K_{J}^{*}\right\}_{1}^{n+1}$ is strictly increasing.

(b) Given the chain of filters $\mathscr{G}_{1} \subsetneq \mathscr{G}_{2} \varsubsetneqq \cdots$, choose $I_{j} \in \mathscr{G}_{j+1}-\mathscr{G}_{j}$ for every $j$. Now set $K_{n}=I_{1} \cap \cdots \cap I_{n}$ for every $n$. It easily follows that $\left\{K_{j}\right\}_{1}^{\infty}$ is a strictly decreasing infinite sequence of right ideals.

Notice that the valuation domain $Z_{(p)}$ shows that (a) and (b) might not occur simultaneously: in fact, $\mathbf{K}\left(Z_{(p)}\right)$ contains strictly increasing infinite sequences but no decreasing sequence can be infinite.

In connection with Proposition 20 we now show a ring, obtained by the usual idealization process, with interesting features on its own.

EXAMPLE 3. There exists a ring $R$ having infinitely many right ideals but only a finite number of left ideals, for which the set of filters of right ideals coincides with the set of filters of left ideals. In addition, $\mathbf{K}(R)$ is a finite linearly ordered lattice.

In fact, let $F \subset G$ be a field extension constructed in such a way that $\operatorname{dim}_{F}(G)=n$ and there exists a field isomorphism $f: G \rightarrow F$. Now set $A=\left\|\begin{array}{ll}G & G\end{array}\right\|$ and the subring of $A$ given by $R=\left\{\left(\begin{array}{cc}a & a^{\prime} \\ 0 & b\end{array}\right) \in A ; f(a)=b\right\} . R$ has thus only three left ideals: $(0), R$ and $P=\left\|_{0}^{0}{ }_{0}^{G}\right\|$.

On the other hand, the infinitely many right ideals of $R$ are given by $\left\|\begin{array}{lll}0 & V_{0}\end{array}{ }_{0}\right\|$, where $V$ runs through the $F$-subspaces of $G ; R$ is clearly right artinian and its only ideals are (0), $P$ and $R$. By Proposition 8a, it follows that $\mathbf{K}(R)$ is linearly ordered, and the filters of right ideals of $R$ are $\overline{\mathscr{F}}(0)=\left\{I_{R} ; I \subset R\right\}, \overline{\mathscr{F}}(R)=\{R\}$ and $\overline{\mathscr{F}}(P)=\{P, R\}$. Hence, $\mathbf{K}(R)$ $=\{\mathbf{0}$, Soc,$\infty\}$ and $\sigma_{\max }=$ Soc which is not an idempotent kernel since $P^{2} \neq P$. 
We next refer again to Proposition 8: this time, however, with the corresponding second statement applied to the category of left modules, and infer that $R$ admits only three filters of left ideals, which coincide with those we found above. $R$ satisfies therefore the required properties. It is worth noticing that the filter associated with $\sigma_{\max }$ does not coincide with $\left\{I_{R} ; I \neq(0)\right\}$, a fact anticipated in the comments preceding Proposition 12.

The following example exhibits a simple domain $R$, not a field, such that $\mathbf{K}(R)$ is linearly ordered.

EXAMPLE 4. There exists a non-artinian simple principle ideal domain $R$ for which $\mathbf{K}(R)=\mathbf{I}(R)$ has exactly three elements.

In fact, set $R=K[x ; D]$ the ring of differential polynomials over a Kolchin universal field $(K, D)$. It is known $R$ has the required ring structure. There is a unique simple $R$-module $S$ (up to isomorphisms); $S$ is, in fact, injective. In addition, if $M_{R}$ is cyclic, then either $M \cong R$ or $M$ is semisimple, which implies $R$ is a PCI-ring. (See [2], Thm. 7.42.) It follows easily that $\mathbf{K}(R)=\{\mathbf{0}$, Soc, $\infty\}$. Moreover, since every semisimple $R$-module is injective, $\operatorname{Soc}(M / \operatorname{Soc}(M))=(0)$ for every $M$ and therefore $\mathbf{K}(R)=\mathbf{I}(R)$ as claimed. Notice that $\sigma_{\max }=\mathrm{Soc}=Z$, and its associated filter coincides with $\left\{I_{R} ;(0) \neq I \subset R\right\}$.

The examples given show that " $\mathbf{K}(R)$ linearly ordered" can be thought of as a unifing concept for rings with quite different structures.

A Semigroup Structure on $\mathbf{K}(R)$. For any given ring $R, \mathbf{K}(R)$ can be given a semigroup structure in the following way: given $\sigma$ and $\lambda$ in $\mathbf{K}(R)$ let $\mathscr{A}$ and $\mathscr{B}$ be their corresponding torsion classes. Consider the collection $\mathscr{C}=\left\{M \in \mathscr{M}_{R}\right.$; there exists an exact sequence $(0) \rightarrow A \rightarrow M \rightarrow B$ $\rightarrow(0)$ with $A \in \mathscr{A}$ and $B \in \mathscr{B}\}$. It is routine checking that $\mathscr{C}$ is a torsion class. Denote by $\sigma * \lambda$ the corresponding kernel functor. It is easily verified that $*$ is associative and so $(\mathbf{K}(R), *)$ is a semigroup. Some basic properties of this semigroup are summarized next:

For all $\nu \in \mathbf{K}(R)$

(a) $\mathbf{0} * \nu=\nu * \mathbf{0}=\nu$.

(b) $\infty * \nu=\nu * \infty=\infty$.

(c) If $\sigma_{1} \leq \sigma_{2}$, then $\sigma_{1} * \nu \leq \sigma_{2} * \nu$ and $\nu * \sigma_{1} \leq \nu * \sigma_{2}$.

(d) $\nu \leq \nu * \nu$; and $\nu=\nu * \nu$ if and only if $\nu \in \mathbf{I}(R)$.

(e) $\sigma \leq \sigma * \nu$ and $\nu \leq \sigma * \nu$ for all $\sigma \in \mathbf{K}(R)$.

(f) If $\nu \leq \sigma$ and $\sigma \in \mathbf{I}(R)$ then $\nu * \sigma=\sigma$. 
Notice that $(\mathbf{K}(R), *)$ is never a group, since $\sigma * \nu=\mathbf{0}$ implies $\sigma=\nu$ $=\mathbf{0}$.

The following example shows that $(\mathbf{K}(R), *)$ may not be commutative.

EXAMPLE 5. If $R$ is the ring in Example 2 we claim that $\operatorname{Soc} * \sigma_{\max }=$ $\sigma_{\max }$ and $\sigma_{\max } * \operatorname{Soc}=\infty$. In fact, if Soc $* \sigma_{\max } \neq \sigma_{\max }$ by (e) we must have Soc $* \sigma_{\max }>\sigma_{\max }$ and so Soc $* \sigma_{\max }=\infty$. There exists then an exact sequence $(0) \rightarrow X \rightarrow R \rightarrow M \rightarrow(0)$ for a certain Soc-torsion module $X$ and $\sigma_{\text {max }}$-torsion module $M$. Therefore $R$ contains non-zero minimal ideals, a contradiction.

On the other hand, in order to prove that $\sigma_{\max } *$ Soc $=\infty$ it suffices to show that $R_{R}$ is $\nu$-torsion, where $\nu=\sigma_{\max } *$ Soc. To that end, consider the exact sequence $(0) \rightarrow A_{0} \rightarrow R \rightarrow R / A_{0} \rightarrow(0)$.

For every $a \in A_{0},(0: a)$ is large in $R$ and therefore $a \in Z(R)=$ $\sigma_{\text {max }}(R)$. It follows that $A_{0}$ is a $\sigma_{\text {max }}$-torsion module. Also, since $R / A_{0}$ is a simple module, it is Soc-torsion. We conclude that $R$ is a $\nu$-torsion module.

However, if $\mathbf{K}(R)$ is linearly ordered and $\mathbf{K}(R)=\mathbf{I}(R)$ then $(\mathbf{K}(R), *)$ is a commutative semigroup (use c, e and f). As in [8] it is therefore valid to try to determine when $(\mathbf{K}(R), *)$ is the valuation semigroup of a valuation ring.

Let us recall that given a valuation ring $A$, its valuation semigroup is defined as the set $A^{*}=\{a A ; a \in A\}$ together with the binary operation $a A+b A=a b A$. Let $a^{*}$ denote $a A$; therefore $A^{*}$ is a commutative semigroup whose identity element is $1^{*}$. If we define $a^{*} \leq b^{*}$ if and only if $a A \supset b A, A^{*}$ turns out to be linearly ordered, with minimum $1^{*}$ and maximum $0^{*}$. Assume $\mathbf{K}(R)=A^{*}$ and pick $\nu$ in $\mathbf{K}(R)-\{\mathbf{0}\}$; it corresponds to a certain $a^{*} \in A^{*}-\left\{1^{*}\right\}$. According to (d) $\nu * \nu=\nu$, and thus $a^{*}+a^{*}=a^{*}$, that is, $a^{2} A=a A$. There exists $x \in A$ such that $a=a^{2} x$ and since $1-a x$ is a unit, $a$ must equal zero. We obtain $a^{*}=0^{*}$ and consequently $\nu=\infty$. Conclude that $\mathbf{K}(R)=\{\mathbf{0}, \infty\}$, that is, $R \cong$ $M_{n \times n}(D)$, for a certain division ring $D$.

We summarize this section by stating the following

THEOREM 21. Assume $\mathbf{K}(R)$ is linearly ordered and $\mathbf{K}(R)=\mathbf{I}(R)$. Then, $(\mathbf{K}(R), *)$ is the valuation semigroup of a valuation ring if and only if $R \cong M_{n \times n}(D)$ where $n$ is a natural number and $D$ is a division ring. 


\section{REFERENCES}

[1] H. H. Brungs, Semigroups associated with right chain rings, Comm. Algebra, 8 (1980), 79-85.

[2] C. Faith, Algebra: Rings, Modules and Categories I, Springer-Verlag, NY (1973).

[3] _ Algebra II: Ring Theory, Springer-Verlag, NY (1976).

[4] O. Goldman, Rings and modules of quotients, J. Algebra, 13 (1969), 10-47.

[5] J. Ohm and P. Vicknair, Monoid rings which are valuation rings, Comm. in Algebra, 11 (12), (1983), 1355-1368.

[6] B. Osofsky, On twisted polynomial rings, J. Algebra, 18 (1971), 597-607.

[7] R. Rubin, Absolutely torsion free rings, Pacific J. Math., 2 (1973), 503-514.

[8] T. S. Shores, On generalized valuation rings, Michigan Math. J., 21 (1974), 405-409.

[9] B. Stenstrom, Rings and Modules of Quotients, Lecture Notes in Mathematics, vol. 237, Springer-Verlag (1971).

[10] J. Viola-Prioli, On absolutely torsion free rings and kernel functors, Pacific J. Math., 56 (1975), 275-283.

[11] __, When is every kernel functor idempotent? Canad. J. Math., 27 (1975), 545-554.

Received October 15, 1986.

UNIVERSIDAD SIMON BOLIVAR

Caracas, Venezuela 


\section{PACIFIC JOURNAL OF MATHEMATICS \\ EDITORS}

V. S. VARADARAJAN

(Managing Editor)

University of California

Los Angeles, CA 90024

Herbert Clemens

University of Utah

Salt Lake City, UT 84112

R. FINN

Stanford University

Stanford, CA 94305
HERMANN FLASCHKA

University of Arizona

Tucson, AZ 85721

RAMESH A. GANGOLLI

University of Washington Seattle, WA 98195

VAUGHAN F. R. JONES

University of California

Berkeley, CA 94720
ROBION KIRBY

University of California

Berkeley, CA 94720

C. C. MOORE

University of California

Berkeley, CA 94720

HAROLD STARK

University of California, San Diego

La Jolla, CA 92093

\section{ASSOCIATE EDITORS}
R. ARENS
E. F. BECKENBACH
B. H. NEUMANN
F. WOLF
K. YOSHIDA
(1906-1982)

\section{SUPPORTING INSTITUTIONS}

UNIVERSITY OF ARIZONA

UNIVERSITY OF BRITISH COLUMBIA

CALIFORNIA INSTITUTE OF TECHNOLOGY

UNIVERSITY OF CALIFORNIA

MONTANA STATE UNIVERSITY

UNIVERSITY OF NEVADA, RENO

NEW MEXICO STATE UNIVERSITY

OREGON STATE UNIVERSITY
UNIVERSITY OF OREGON UNIVERSITY OF SOUTHERN CALIFORNIA

STANFORD UNIVERSITY

UNIVERSITY OF HAWAII

UNIVERSITY OF TOKYO

UNIVERSITY OF UTAH

WASHINGTON STATE UNIVERSITY

UNIVERSITY OF WASHINGTON 


\section{Pacific Journal of Mathematics \\ Vol. 132, No. $1 \quad$ January, 1988}

Scott W. Brown, Full analytic subspaces for contractions with rich spectrum ...1

Robert Main Burton, Jr. and Tae-Sung Kim, An invariance principle for

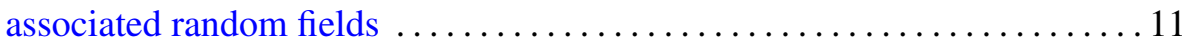

Ana M. Viola-Prioli and Jorge Viola-Prioli, Rings whose kernel functors

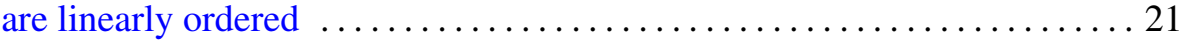

David E. Handelman, Representing polynomials by positive linear

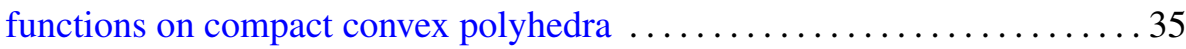

Patrick Keef, On the Tor functor and some classes of abelian groups . .....66

Dennis R. Malm, Simplicity of partial and Schmidt differential operator

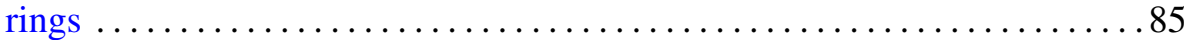

José M. Montesinos and Carmen Safont, On the Birman invariants of

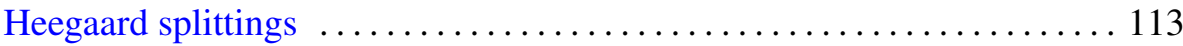

N. P. Mukherjee and Prabir Bhattacharya, The normal index of a finite group

Mario Petrich and Norman R. Reilly, Semigroups generated by certain operators on varieties of completely regular semigroups

Robert Tijdeman and Lian Xiang Wang, Sums of products of powers of given prime numbers

Joel Larry Weiner, First integrals for a direction field on a simply connected plane domain 